\title{
Global non-existence for some nonlinear wave equations with damping and source terms in an inhomogeneous medium
}

Yanjin Wang ${ }^{1 *}$ and Yufeng Wang ${ }^{2}$

\section{"Correspondence:}

wang_yanjin@iapcm.ac.cn ${ }^{1}$ Institute of Applied Physics and Computational Mathematics, No. 2, Fenghaodonglu, District Haidian, Beijing, 100094, China

Full list of author information is available at the end of the article

\begin{abstract}
In this paper we study the initial value problem for the nonlinear wave equation with damping and source terms

$$
u_{t t}-\rho(x)^{-1} \Delta u+u_{t}+m^{2} u=f(u)
$$

with some $\rho(x)$ and $f(u)$ on the whole space $\mathbb{R}^{n}(n \geq 3)$.

For the low initial energy case, which is the non-positive initial energy, based on a concavity argument we prove the blow-up result. As for the high initial energy case, we give sufficient conditions of the initial data such that the corresponding solution blows up in finite time. In other words, our results imply a complete blow-up theorem in the sense of the initial energy, $-\infty<E(0)<+\infty$.
\end{abstract}

MSC: 35L15; 35Q72

Keywords: wave equations; blow-up; high initial energy; damping term; inhomogeneous medium

\section{Introduction}

In this paper our aim is to study some nonlinear wave equation with damping and source terms in the following form:

$$
\begin{cases}u_{t t}-\rho(x)^{-1} \Delta u+u_{t}+m^{2} u=f(u), & (t, x) \in[0, T) \times \mathbb{R}^{n}, \\ u(0, x)=u_{0}(x), & x \in \mathbb{R}^{n}, \\ u_{t}(0, x)=u_{1}(x), & x \in \mathbb{R}^{n},\end{cases}
$$

where $\Delta$ is Laplacian operator on $\mathbb{R}^{n}(n \geq 3), u_{0}(x)$ and $u_{1}(x)$ are real valued functions, $m$ is a real constant (the case $m=0$ is called the mass free case; $m \neq 0$ the mass case), $\rho(x)$ satisfies the following condition:

(H) $\rho(x)>0$ for every $x \in \mathbb{R}^{n}, \rho \in \mathcal{C}^{0, \gamma}\left(\mathbb{R}^{n}\right)$ with $\gamma \in(0,1)$, and $\rho \in L^{n / 2}\left(\mathbb{R}^{n}\right) \cap L^{\infty}\left(\mathbb{R}^{n}\right)$.

The wave equations (1) appear in applications in various areas of mathematical physics (see, for example, [1-3]), as well as in geophysics and ocean acoustics, where, for example, the coefficient $\rho(x)$ represents the speed of sound at the point $x \in \mathbb{R}^{N}$ (see [4]), in other 
words, $\rho(x) \neq$ constant implies that the medium where the sound travels is inhomogeneous.

For the nonlinear power, throughout the paper we make the following assumption: for the nonlinear power $f(s)$ there exists some constant $\epsilon>0$ such that

$$
f(s) s \geq(2+\epsilon) F(s)
$$

for any $s \in \mathbb{R}$, where

$$
F(\zeta)=\int_{0}^{\zeta} f(\kappa) d \kappa
$$

The nonlinear power satisfying (2) was firstly introduced for abstract wave equations with $\rho(x)=1$ by Levine [5]. And then Cazenave [6] also considered it for Klein-Gordon equations.

Before going any further, we briefly introduce some results for the wave equation (1) with $\rho(x)=$ constant $\neq 0$ (without loss of generality let $\rho(x)=1$ ), obviously it does not satisfy the assumption $(\mathrm{H})$. The general nonlinear power $f(u)$ with (2) it was firstly considered for some abstract wave equations in [5], where Levine proved the blow-up result when the initial energy was negative. But mostly the results as regards the Cauchy problem of the wave equation were established for the typical form of nonlinear power as

$$
f(u)=|u|^{p-1} u,
$$

where $1<p<\frac{n+2}{n-2}$ as $n \geq 3$ and $1<p<\infty$ as $n=1,2$. Here we note that the above power satisfies the condition (2). For the power (4), the wave equations with damping term were studied by many authors. It is well known that the local solution blows up in finite time when the initial energy is negative. For global existence and non-existence of solutions for the Cauchy problem of equation (1) with $\rho(x)=1,(4)$ and a (possibly nonlinear) damping term, we here refer to [7-9]. In particular, recently the wave equation with damping term was considered in [10], where Levine and Todorova showed that for arbitrarily positive initial energy there are choices of initial data such that the local solution blows up in finite time. Subsequently, Todorova and Vitillaro [11] established more precise result as regards the existence of initial values such that the corresponding solution blows up in finite time for arbitrarily high initial energy. More recently, Gazzola and Squassina [12] established sufficient conditions of initial data with arbitrarily positive initial energy such that the corresponding solution blows up in finite time for the wave equation with linear damping and (4) in the mass free case on a bounded Lipschitz subset of $\mathbb{R}^{n}$. The first author [13] also studied the blow-up result for the Klein-Gordon equation with arbitrarily positive initial energy. Also the authors [14] investigated the non-existence of global solutions for some coupled Klein-Gordon equations with arbitrary initial energy. Also, the wave equations have been investigated with some special boundary conditions [15].

For the case $\rho(x)=1$ and $m=0$, we also note that the equations were also considered with $f(u)=|u|^{p}$ by some authors. Here we just refer to [16] and the references therein.

Now we return to equation (1) with some general $\rho(x)$. For the linear case, $f(u)=0$, Eidus [17] first studied the existence of solutions for the linear wave equation (1). Then Karachalios and Stavrakakis [18] studied the existence of the solution of the damped wave 
equation (1) with some nonlinear power. And they [19] also established the results as regards the global existence and blow-up of solutions for equation (1) with $(\mathrm{H})$ and (4) in the free mass case by the potential well method, which was firstly developed by Sattinger [20]. Their blow-up result was under the condition that the initial energy was negative. Recently, for equation (1) with (H) and (4) Zhou [21] investigated the global existence and blow-up result including the mass free case and mass case. Zhou established the blow-up result when the initial energy was less than a positive constant, which is independent from the initial data. But in all the work mentioned above, equation (1) with high initial energy was not considered under the assumption $(\mathrm{H})$.

The main purpose of this paper is to establish the blow-up result for equation (1) with $(\mathrm{H})$ and $(2)$ when the initial energy is high. Based on a concavity argument, which was used to establish blow-up of solutions to nonlinear damped wave equations (see e.g. [21, 22 ] and the references therein), we firstly establish the blow-up result when the initial energy is non-positive. Because of the nonlinearity, $\rho(x)$ is not constant. We cannot directly apply the proof method of $[13,14]$ for equations $(1)$ in this paper. Note that, when the initial energy is zero, the blow-up result was also established in [21], where the assumption is needed that $\rho(x) \in L^{1}\left(\mathbb{R}^{n}\right)$ with $(\mathrm{H})$ and $\int \rho(x) u_{0}(x) u_{1}(x) d x \geq 0$. Thus in some sense (see Remark 1 ) we improve the blow-up result in [21]. As for the case with the arbitrarily positive initial energy, we establish the blow-up result for (1) under some conditions of $\left(u_{0}, u_{1}\right)$ on the whole space $\mathbb{R}^{n}$. To the best of our knowledge this is the first blow-up result with high initial energy for equation (1) with $(\mathrm{H})$.

Moreover, we note that if $\rho(x)$ satisfies $(\mathrm{H})$ then the mass $m$ does not affect the blow-up result, but if $\rho=$ constant $\neq 0$ it will affect the blow-up result, that is, if $m=0$ and $\rho(x)=1$ then the blow-up result is obtained only on a bounded subset of $\mathbb{R}^{n}$. Indeed, by $(\mathrm{H})$ we see that $\rho(x)$ will be a rapidly enough decreasing at infinite time, thus it makes it possible to consider equation (1) on the whole space $\mathbb{R}^{n}$ in the mass free case.

The paper is composed of three sections. In the next section we introduce some notations and well-known results, and we also state our main results. In the last section we prove our results.

\section{Principal results}

In order to state our main results, we briefly mention some facts, notations, and wellknown results. We denote by $\|\cdot\|_{q}$ the $L^{q}\left(\mathbb{R}^{n}\right)$ norm for $1 \leq q \leq \infty$, and we define the functional spaces: $H^{1}\left(\mathbb{R}^{n}\right)=\left\{u \in L^{2}\left(\mathbb{R}^{n}\right) ;\|u\|_{H^{1}\left(\mathbb{R}^{n}\right)}=\left\|(1-\Delta)^{1 / 2} u\right\|<\infty\right\}$, and $H_{0}^{1}\left(\mathbb{R}^{n}\right)=\{u \in$ $H^{1}\left(\mathbb{R}^{n}\right)$; $\operatorname{supp}(u)$ is compact in $\mathbb{R}^{n}$. For simplicity we will denote $\int_{\mathbb{R}^{n}}$ by $\int$. The notation $t \rightarrow T^{-}$means $t<T$ and $t \rightarrow T$.

As in [23], we introduce the function space $X^{1,2}\left(R^{n}\right)$, which is defined as the closure of $C_{0}^{\infty}$ functions with respect to the energy norm $\|u\|_{X^{1,2}}:=\int|\nabla u(x)|^{2} d x$, that is,

$$
X^{1,2}\left(\mathbb{R}^{n}\right)=\left\{u \in L^{\frac{2 n}{n-2}}\left(\mathbb{R}^{n}\right): \nabla u \in\left(L^{2}\left(\mathbb{R}^{n}\right)\right)^{n}\right\} .
$$

It is well known that $X^{1,2}$ is embedded continuously in $L^{\frac{2 n}{n-2}}$, which means that there exists a constant $k>0$ such that

$$
\|u\|_{\frac{2 n}{n-2}} \leq k\|u\|_{X^{1,2}} .
$$

Following (6), we have the following inequality (see [24]). 
Lemma 1 Suppose $\rho \in L^{\frac{n}{2}}\left(\mathbb{R}^{n}\right)$ and $n \geq 3$. Then there exists a constant $\alpha>0$ such that

$$
\int|\nabla u(x)|^{2} d x \geq \alpha \int \rho(x)|u(x)|^{2} d x
$$

for every $u \in C_{0}^{\infty}\left(\mathbb{R}^{n}\right)$. Moreover, $\alpha=k^{-2}\|\rho\|_{\frac{n}{2}}^{-1}$ where $k$ is defined in (6).

In addition, the weighted space $L_{\rho}^{2}\left(\mathbb{R}^{n}\right)$ is defined to be the closure of $C_{0}^{\infty}\left(\mathbb{R}^{n}\right)$ functions with respect to the inner product

$$
(u, v)_{L_{\rho}^{2}}:=\int \rho(x) u(x) v(x) d x
$$

and norm

$$
\|u\|_{L_{\rho}^{2}}^{2}=(u, u)_{L_{\rho}^{2}}
$$

For the local existence of solutions of equation (1), we state the following.

Theorem 1 Under the assumption $(\mathrm{H})$, for any initial data $\left(u_{0}, u_{1}\right) \in X^{1,2}\left(\mathbb{R}^{n}\right) \times L_{\rho}^{2}\left(\mathbb{R}^{n}\right)$, if $f$ satisfies the conditions $f(0)=0$ and

$$
\left|f\left(\lambda_{1}\right)-f\left(\lambda_{2}\right)\right| \leq c\left(\left|\lambda_{1}\right|^{p-1}+\left|\lambda_{2}\right|^{p-1}\right)\left|\lambda_{1}-\lambda_{2}\right|
$$

for all $\lambda_{1}, \lambda_{2} \in \mathbb{R}$, some constant $c>0$, and

$$
1<p<\frac{n}{n-2} \quad \text { when } n \geq 3
$$

then there exists a unique local solution $u(t)$ of the equation (1) on a maximal time interval $\left[0, T_{\max }\right)$ satisfying

$$
\begin{aligned}
& u \in C\left(\left[0, T_{\max }\right) ; X^{1,2}\left(\mathbb{R}^{n}\right)\right) \text { and } u_{t} \in C\left(\left[0, T_{\max }\right) ; L_{\rho}^{2}\right), \\
& u(0, x)=u_{0}(x) \text { and } u_{t}(0, x)=u_{1}(x) .
\end{aligned}
$$

In addition, $u(t)$ satisfies

$$
E(0)-E(t)=\int_{0}^{t}\left\|u_{\tau}(\tau, \cdot)\right\|_{L_{\rho}^{2}}^{2} d \tau \geq 0
$$

for every $t \in\left[0, T_{\max }\right)$, where

$$
\begin{aligned}
E(t)= & \frac{1}{2} \int\left(\rho(x)\left|u_{t}(t, x)\right|^{2}+|\nabla u(t, x)|^{2}\right. \\
& \left.+m^{2} \rho(x)|u(t, x)|^{2}-2 \rho(x) F(u(t, x))\right) d x,
\end{aligned}
$$

where $F(u)$ is defined in (3). 
This result can be proved by the Banach fixed point theorem. The proof follows from the weighted-norm Lebesgue space of the corresponding theorem for the wave equations of Kirchhoff type [25].

If $T_{\max }<\infty$, then the local solution is said to blow up in finite time $T_{\max }$. Otherwise, $T_{\max }=\infty$, the corresponding local solution is global.

Next we state our first blow-up result for equation (1) with (H) and (2) in the case of non-positive initial energy.

Theorem 2 Under the assumptions $(\mathrm{H})$ and $(2)$, if the nonzero initial data $\left(u_{0}, u_{1}\right) \in$ $X^{1,2}\left(\mathbb{R}^{n}\right) \times L_{\rho}^{2}\left(\mathbb{R}^{n}\right)$ satisfies

$$
E(0)<0,
$$

or

$$
\int \rho(x) u_{0}(x) u_{1}(x) d x \geq 0 \quad \text { if } E(0)=0,
$$

then the corresponding local solution of equation (1) blows up in finite time, $T_{\max }<\infty$.

Remark 1 In the case $E(0)<0$, Zhou [21] also established the blow-up result for equation (1) with $(\mathrm{H})$ and (4) under the further assumptions $\int \rho(x) u_{0}(x) u_{1}(x) d x \geq 0$ and $\rho \in L^{1}\left(\mathbb{R}^{n}\right)$. But in the above theorem, we remove $\rho \in L^{1}\left(\mathbb{R}^{n}\right)$. Thus, in this sense we improve the result [21].

To state our main blow-up result with arbitrarily positive initial energy, we introduce a function as follows:

$$
I(u)=\int\left(|\nabla u(x)|^{2}+m^{2} \rho(x)|u(x)|^{2}-\rho(x) f(u(x)) u(x)\right) d x .
$$

Now we introduce our main blow-up result for equation (1) with arbitrarily positive initial energy, as far as we know, which is the first blow-up result for equation (1) with (H) on the whole space $\mathbb{R}^{n}$.

Theorem 3 Under the assumptions $(\mathrm{H})$ and $(2)$, if the initial data $\left(u_{0}, u_{1}\right) \in X^{1,2}\left(\mathbb{R}^{n}\right) \times$ $L_{\rho}^{2}\left(\mathbb{R}^{n}\right)$ satisfies

$$
\begin{aligned}
& E(0)>0, \\
& I\left(u_{0}\right)<0, \\
& \int \rho(x) u_{0}(x) u_{1}(x) d x \geq 0, \\
& \left\|u_{0}\right\|_{L_{\rho}^{2}}^{2}>\frac{2(2+\epsilon)}{m^{2} \epsilon} E(0) \quad \text { when } m \neq 0, \\
& \left\|u_{0}\right\|_{L_{\rho}^{2}}^{2}>\frac{2(2+\epsilon)}{\min \{1, \alpha\} \epsilon} E(0) \quad \text { when } m=0,
\end{aligned}
$$

where $\epsilon$ and $\alpha$ are stated in (2) and Lemma 1, respectively, then the corresponding local solution of equation (1) blows up in finite time $T_{\max }<\infty$. 
Remark 2 We note that, for the case $E(0)<0$, by (15) and $(14), I(u(t, \cdot))<0$ for every $t \in\left[0, T_{\max }\right)$.

Reading Theorems 2, 3 and Remark 2, naturally one considers the local solution when the initial data satisfies $E(0)>0$ and $I\left(u_{0}\right)>0$. Indeed, for this case, being similar to the argument with $m=0$ and $f(u)=|u|^{p-1} u$ in [19], by a potential well method we can also obtain the global existence of solutions of equation (1) with $(\mathrm{H})$ and $(2)$ when the positive initial energy is small enough. Here we omit it. Furthermore, it is still open whether there exists a global solution for wave equations when the initial energy is arbitrarily high.

\section{Proof of the main theorems}

In this section, we prove Theorems 2 and 3 based on a concavity argument. Firstly, we introduce a lemma concerned with the concavity argument [5].

Lemma 2 Let $F(t) \in C^{2}([0, T))$ be a function satisfying that

$$
F F^{\prime \prime}-(\alpha+1)\left(F^{\prime}\right)^{2} \geq 0
$$

for some $\alpha>0$, if $F(0)>0$ and $F^{\prime}(0)>0$ then $F(t) \rightarrow+\infty$ as $t \rightarrow T_{\max }\left(\leq F(0) / \alpha F^{\prime}(0)\right)$.

We next show a lemma, which plays a role in our proofs of Theorems 2 and 3.

Lemma 3 Assume that $\left(u_{0}, u_{1}\right) \in X^{1,2}\left(\mathbb{R}^{n}\right) \times L_{\rho}^{2}\left(\mathbb{R}^{n}\right)$ satisfies

$$
\int \rho(x) u_{0}(x) u_{1}(x) d x \geq 0
$$

If the corresponding local solution $\left(u(t, x), u_{t}(t, x)\right) \in C\left(\left[0, T_{\max }\right), X^{1,2}\left(\mathbb{R}^{n}\right) \times L_{\rho}^{2}\left(\mathbb{R}^{n}\right)\right)$ is such that

$$
I(u(t, \cdot))<0
$$

for every $t \in\left[0, T_{\max }\right)$, then $\|u(t, \cdot)\|_{L_{\rho}^{2}}^{2}$ is strictly increasing on $\left[0, T_{\max }\right)$.

Proof Since $u(t, x)$ is the local solution of equation (1), we easily have

$$
\begin{aligned}
\frac{1}{2} \frac{d^{2}}{d t^{2}} \int \rho(x)|u(t, x)|^{2} d x= & \int \rho(x)\left|u_{t}(t, x)\right|^{2} d x+\int \rho(x) u(t, x) u_{t}(t, x) \\
& -\int\left(m^{2} \rho(x)|u(t, x)|^{2}+|\nabla u(t, x)|^{2}\right. \\
& -\rho(x) u(t, x) f(u(t, x))) d x
\end{aligned}
$$

for every $t \in\left[0, T_{\max }\right)$.

As a result, it follows that

$$
\frac{d^{2}}{d t^{2}} \int \rho(x)|u(t, x)|^{2} d x+\frac{d}{d t} \int \rho(x)|u(t, x)|^{2} d x=2\left(\left\|u_{t}(t, \cdot)\right\|_{L_{\rho}^{2}}^{2}-I(u(t, \cdot))\right) .
$$


By (26) and the above equation we have

$$
\frac{d^{2}}{d t^{2}} \int \rho(x)|u(t, x)|^{2} d x+\frac{d}{d t} \int \rho(x)|u(t, x)|^{2} d x>0
$$

for every $t \in\left[0, T_{\max }\right)$.

Here we let $H(t)=\frac{d}{d t} \int \rho(x)|u(t, x)|^{2} d x$, then as in [19] we see that the function $H(t)$ is a Lipschitzian function over $\left[0, T_{\max }\right)$. Thus, by $(28)$ it follows that $\|u(t, \cdot)\|_{L_{\rho}^{2}}^{2}$ is strictly increasing on $\left[0, T_{\max }\right)$.

Proof of Theorem 2 We first define the following auxiliary function:

$$
\begin{aligned}
G(t)= & \int \rho(x)|u(t, x)|^{2} d x+\int_{0}^{t}\|u(\tau, \cdot)\|_{L_{\rho}^{2}}^{2} d \tau \\
& +\left(T_{0}-t\right)\left\|u_{0}\right\|_{L_{\rho}^{2}}^{2}+\zeta\left(T_{1}+t\right)^{2}
\end{aligned}
$$

where the constants, $T_{0}>0, T_{1}>0, \zeta>0$, will be determined later.

We then have

$$
\begin{aligned}
G^{\prime}(t)= & \frac{d}{d t} G(t) \\
= & 2 \int \rho(x) u(t, x) u_{t}(t, \cdot) d x+\|u(t, \cdot)\|_{L_{\rho}^{2}}^{2}-\left\|u_{0}\right\|_{L_{\rho}^{2}}^{2}+2 \zeta\left(T_{1}+t\right) \\
= & 2 \int \rho(x) u(t, x) u_{t}(t, \cdot) d x+2 \int_{0}^{t}\left(u(\tau, \cdot), u_{\tau}(\tau, \cdot)\right)_{L_{\rho}^{2}} d \tau \\
& +2 \zeta\left(T_{1}+t\right)
\end{aligned}
$$

and

$$
\begin{aligned}
\frac{1}{2} G^{\prime \prime}(t)= & \int \rho(x)\left|u_{t}(t, x)\right|^{2} d x+\int \rho(t, x) u(t, x) u_{t t}(t, x) d x \\
& +\int \rho(x) u(t, x) u_{t}(t, x) d x+\zeta \\
= & \int \rho(x)\left|u_{t}(t, x)\right|^{2} d x+\int \rho(x) u(t, x) f(u(t, x)) d x \\
& -\int\left(|\nabla u(t, x)|^{2}+m^{2} \rho(x)|u(t, x)|^{2}\right) d x+\zeta .
\end{aligned}
$$

Case I: $E(0)<0$. By (2), (14), and (15), we see that

$$
\begin{aligned}
\int \rho(x) F(u(t, x)) d x= & \frac{1}{2} \int\left(m^{2} \rho(x)|u(t, x)|^{2}+\rho(x)\left|u_{t}(t, x)\right|^{2}+|\nabla u(t, x)|^{2}\right) d x \\
& -E(0)+\int_{0}^{t}\left\|u_{\tau}(\tau, x)\right\|_{L_{\rho}^{2}}^{2} d \tau \\
\leq & \frac{1}{2+\epsilon} \int \rho(x) u(t, x) f(u(t, x)) d x
\end{aligned}
$$

for all $t \in\left[0, T_{\max }\right)$. 
Thus, from (31) it follows that

$$
\begin{aligned}
G^{\prime \prime}(t) \geq & (4+\epsilon) \int \rho(x)\left|u_{t}(t, x)\right|^{2} d x+2(2+\epsilon) \int_{0}^{t}\left\|u_{\tau}(\tau, \cdot)\right\|_{L_{\rho}^{2}}^{2} d \tau \\
& +\epsilon \int\left(|\nabla u(t, x)|^{2}+m^{2} \rho(x)|u(t, x)|\right) d x-2(2+\epsilon) E(0)+2 \zeta
\end{aligned}
$$

We now let the constant $\zeta$ satisfy

$$
0<\zeta \leq-2 E(0)
$$

Then it follows from (34)

$$
-2(2+\epsilon) E(0)+2 \zeta \geq(4+\epsilon) \zeta
$$

Obviously, $G^{\prime \prime}(t)>0$ on $\left[0, T_{\max }\right)$. Moreover, we can take $T_{1}>0$ sufficiently large such that

$$
G^{\prime}(0)=2 \int \rho(x) u_{0}(x) u_{1}(x)+2 B T_{1}>0
$$

and

$$
\frac{\epsilon}{2}\left(\int \rho(x) u_{0}(x) u_{1}(x) d x+\zeta T_{1}\right)>\int \rho(x)\left|u_{0}(x)\right|^{2} d x
$$

Thus, by (36) we see that $G(t)>0, G^{\prime}(t)>0$, and $G^{\prime \prime}(t)>0$ for every $t \in\left[0, T_{\max }\right)$. That is, $G(t)$ and $G^{\prime}(t)$ are strictly increasing on $\left[0, T_{\max }\right)$. Then we let

$$
\begin{aligned}
& A=\int \rho(x)|u(t, x)|^{2} d x+\int_{0}^{t}\|u(\tau, \cdot)\|_{L_{\rho}^{2}}^{2} d \tau+\zeta\left(T_{1}+t\right)^{2}, \\
& B=\frac{1}{2} G^{\prime}(t), \\
& C=\int \rho(x)\left|u_{t}(t, x)\right|^{2} d x+\int_{0}^{t}\left\|u_{\tau}(\tau, \cdot)\right\|_{L_{\rho}^{2}}^{2} d \tau+\zeta .
\end{aligned}
$$

For every $t \in\left[0, T_{0}\right]$ we obviously have

$$
G(t) \geq A,
$$

and by (33) and (35)

$$
G^{\prime \prime}(t) \geq(4+\epsilon) C
$$

We now let $T_{0}$ be sufficiently large and satisfy

$$
T_{0} \geq \frac{4 G(0)}{\epsilon G^{\prime}(0)}
$$

Noting the inequalities (29), (30), and (37), we see that $T_{0}$, defined by (43), is reasonable. 
And suppose the solution $u(t, x)$ exists on $\left[0, T_{0}\right]$, then it follows that

$$
G^{\prime \prime} G(t)-\frac{4+\epsilon}{4}\left(G^{\prime}(t)\right)^{2} \geq(4+\epsilon)\left(A C-B^{2}\right)
$$

for every $t \in\left[0, T_{0}\right]$

By a simple computation we see that

$$
\begin{aligned}
A s^{2}-2 B s+C= & \int \rho(x)\left(s u(t, x)+u_{t}(t, x)\right)^{2} d x \\
& +\int_{0}^{t}\left\|s u(\tau, \cdot)+u_{\tau}(\tau, \cdot)\right\|_{L_{\rho}^{2}}^{2} d \tau+\zeta\left(s\left(T_{1}+t\right)+1\right)^{2} \\
\geq & 0
\end{aligned}
$$

for every $s \in \mathbb{R}$ and $t \in\left[0, T_{0}\right]$, which means that $(2 B)^{2}-4 A C \leq 0$.

Thus we see that

$$
G^{\prime \prime} G(t)-\frac{4+\epsilon}{4}\left(G^{\prime}(t)\right)^{2} \geq 0 .
$$

Since $\frac{4+\epsilon}{4}>1$, we put $\alpha=\frac{\epsilon}{4}$. By Lemma 2 we see that

$$
\lim _{t \rightarrow T_{\max }^{-}}\|u(t, \cdot)\|_{L_{\rho}^{2}}^{2}=\infty
$$

which implies that the corresponding solution $u(t, x)$ of equation (1) blows up in finite time $T_{\max }<\infty$.

Case II: $E(0)=0$ and $\int \rho(x) u_{0}(x) u_{1}(x) d x \geq 0$. By (14) and (15) we have

$$
\int\left(|\nabla u(t, x)|^{2}+m^{2} \rho(x)|u(t, x)|^{2}\right) d x-2 \int \rho(x) F(u(t, x)) d x \leq 0
$$

for every $t \in\left[0, T_{\max }\right)$.

Noting the fact that $\int \rho(x) F(u(t, x)) d x \neq 0$, we obtain by (2)

$$
2 \int \rho(x) F(u(t, x)) d x<\int \rho(x) f(u(t, x)) u(t, x) d x
$$

We then get

$$
I(u(t, x))<0
$$

for every $t \in\left[0, T_{\max }\right)$.

Thus by (17) and Lemma 3 we see that $\|u(t, \cdot)\|_{L_{\rho}^{2}}^{2}$ is strictly increasing on $\left[0, T_{\max }\right)$.

In this case we still use the auxiliary function $G(t)$ as (29).

Thus, according to the proof of Case I, by (17) we see that $G(t)>0, G^{\prime}(t)>0, G^{\prime \prime}(t)>0$ on $\left(0, T_{\max }\right)$, that is to say, $G(t)$ and $G^{\prime}(t)$ are strictly increasing over $\left[0, T_{\max }\right)$. 
And as in (33) we also have

$$
\begin{aligned}
G^{\prime \prime}(t) \geq & (4+\epsilon) \int \rho(x)\left|u_{t}(t, x)\right|^{2} d x+2(2+\epsilon) \int_{0}^{t}\left\|u_{\tau}(\tau, \cdot)\right\|_{L_{\rho}^{2}}^{2} d \tau \\
& +\epsilon \int\left(|\nabla u(t, x)|^{2}+m^{2} \rho(x)|u(t, x)|^{2}\right) d x+2 \zeta \\
\geq & (4+\epsilon) \int \rho(x)\left|u_{t}(t, x)\right|^{2} d x+2(2+\epsilon) \int_{0}^{t}\left\|u_{\tau}(\tau, \cdot)\right\|_{L_{\rho}^{2}}^{2} d \tau \\
& +\epsilon\left(m^{2}+\alpha\right) \int \rho(x)\left|u_{0}(x)\right|^{2} d x+2 \zeta,
\end{aligned}
$$

where the last inequality comes from Lemma 1 and Lemma 3.

Now we let the constant $\zeta$ satisfy

$$
0<\zeta \leq \frac{\epsilon}{2+\epsilon}\left(\alpha+m^{2}\right)\left\|u_{0}\right\|_{L_{\rho}^{2}}^{2}
$$

for the mass free case or the mass case, and the other positive constants, $T_{0}$ and $T_{1}$, be large such that

$$
\begin{aligned}
& T_{0} \geq \frac{4 G(0)}{\epsilon G^{\prime}(0)} \\
& \frac{\epsilon}{2}\left(\int \rho(x) u_{0}(x) u_{1}(x) d x+\zeta T_{1}\right)>\int \rho(x)\left|u_{0}(x)\right|^{2} d x
\end{aligned}
$$

Then by the same argument as Case I, we can claim that the corresponding local solution of equation (1) blows up in finite time.

Thus the proof of Theorem 2 is completed.

In the following part we will address Theorem 3. The next lemma is the crux to prove Theorem 3.

Lemma 4 Under the assumptions on $\rho(x), f(u)$ and $\left(u_{0}, u_{1}\right)$ in Theorem 3 , the corresponding local solution $\left(u(t, x), u_{t}(t, x)\right) \in C\left(\left[0, T_{\max }\right), X^{1,2}\left(\mathbb{R}^{n}\right) \times L_{\rho}^{2}\left(\mathbb{R}^{n}\right)\right)$ satisfies

$$
\begin{aligned}
& I(u(t, \cdot))<0, \\
& \|u(t, \cdot)\|_{L_{\rho}^{2}}^{2}>\frac{2(2+\epsilon)}{m^{2} \epsilon} E(0) \quad \text { when } m \neq 0, \\
& \|u(t, \cdot)\|_{L_{\rho}^{2}}^{2}>\frac{2(2+\epsilon)}{\min \{1, \alpha\} \epsilon} E(0) \text { when } m=0
\end{aligned}
$$

for every $t \in\left[0, T_{\max }\right)$.

Proof Here the proof uses a contradiction argument. We assume that (55) is not true over $\left[0, T_{\max }\right)$, that is, there exists a time $T>0$ such that

$$
T=\min \left\{t \in\left(0, T_{\max }\right) ; I(u(t, \cdot))=0\right\} .
$$


Case I: $m \neq 0$. Since $I(u(t, \cdot))<0$ on $[0, T)$, by Lemma 3 we see that $\|u(t, \cdot)\|_{L_{\rho}^{2}}^{2}$ is strictly increasing over $[0, T)$, which implies that

$$
\|u(t, \cdot)\|_{L_{\rho}^{2}}^{2}>\left\|u_{0}\right\|_{L_{\rho}^{2}}^{2}>\frac{2(2+\epsilon)}{m^{2} \epsilon} E(0)
$$

for $m \neq 0$ and every $t \in(0, T)$.

And by the continuity of $\|u(t, \cdot)\|_{L_{\rho}^{2}}^{2}$ at $t$, we see that

$$
\|u(T, \cdot)\|_{L_{\rho}^{2}}^{2}>\frac{2(2+\epsilon)}{m^{2} \epsilon} E(0)
$$

On the other hand, by (14) and (15) we see that

$$
m^{2}\|u(T, \cdot)\|_{L_{\rho}^{2}}^{2}+\|\nabla u(T, \cdot)\|^{2}-2 \int \rho(x) F(u(T, x)) d x \leq 2 E(T) \leq 2 E(0) .
$$

Moreover, noting the assumption $I(u(T, \cdot))=0$ and (2), we then have

$$
\|u(T, \cdot)\|_{L_{\rho}^{2}}^{2}+\|\nabla u(T, \cdot)\|^{2} \geq(2+\epsilon) \int \rho(x) F(u(T, x)) d x .
$$

Combining (61) and (62) we then obtain

$$
m^{2}\|u(T, \cdot)\|_{L_{\rho}^{2}}^{2}+\|\nabla u(T, \cdot)\|^{2} \leq \frac{2(2+\epsilon)}{\epsilon} E(0) .
$$

Obviously there is a contradiction between (60) and (63). Thus we have proved that

$$
I(u(t, \cdot))<0
$$

for every $t \in\left[0, T_{\max }\right)$.

By Lemma 3 we see that if $I(u(t, \cdot))<0$ for every $t \in\left[0, T_{\max }\right)$, then $\|u(t, \cdot)\|_{L_{\rho}^{2}}^{2}$ is strictly increasing on $t$. Thus, (64) implies that

$$
\|u(t, \cdot)\|_{L_{\rho}^{2}}^{2}>\frac{2(2+\epsilon)}{\epsilon} E(0)
$$

for every $t \in\left[0, T_{\max }\right)$.

Thus the proof for Case I, $m \neq 0$, is complete.

Case II: $m=0$. As in the argument for (60), we can also obtain

$$
\|u(T, \cdot)\|_{L_{\rho}^{2}}^{2}>\frac{2(2+\epsilon)}{\min \{1, \alpha\} \epsilon} E(0) .
$$

Since $m=0$, the inequality (63) is rewritten as

$$
\|\nabla u(T, \cdot)\|^{2} \leq \frac{2(2+\epsilon)}{\epsilon} E(0) .
$$

By Lemma 1, we see that

$$
\alpha\|u(t, x)\|_{L_{\rho}^{2}}^{2} \leq \frac{2(2+\epsilon)}{\epsilon} E(0) .
$$


Thus by $(66)$ and $(68)$ we see that the assumption $I(u(T, \cdot))=0$ is wrong. That is to say, $I(u(t, \cdot))<0$ for every $t \in\left[0, T_{\max }\right)$.

Similarly, we also get

$$
\|u(t, \cdot)\|_{L_{\rho}^{2}}^{2}>\frac{2(2+\epsilon)}{\min \{1, \alpha\} \epsilon} E(0)
$$

for every $t \in\left[0, T_{\max }\right)$.

Thus the proof of Lemma 4 has been completed.

Proof of Theorem 3 Here we still use the auxiliary function G, defined in (29). We have

$$
\begin{aligned}
G^{\prime \prime}(t)= & 2 \int \rho(x)\left|u_{t}(t, x)\right| d x+2 \int \rho(x) f(u(t, x)) u(t x, d x) \\
& -2 \int\left(|\nabla u(t, x)|^{2}+m^{2} \rho(x)|u(t, x)|^{2}\right) d x+2 \zeta \\
= & 2 \int \rho(x)\left|u_{t}(t, x)\right| d x-2 I(u(t, \cdot))+2 \zeta .
\end{aligned}
$$

By Lemma 4, we see that

$$
G^{\prime \prime}(t)>0
$$

for every $t \in\left[0, T_{\max }\right)$.

And from (21) we see that $G^{\prime}(t)>0$ for every $t \in\left(0, T_{\max }\right)$. Thus, it turns out that $G(t)$ and $G^{\prime}(t)$ is strictly increasing on $\left[0, T_{\max }\right)$.

Obviously the inequality (32) is also valid for every $t \in\left[0, T_{\max }\right.$ ). Then by Lemma 1 , Lemma 3, (56), (57), and (70) we have

$$
\begin{aligned}
G^{\prime \prime}(t) \geq & (4+\epsilon) \int \rho(x)\left|u_{t}(t, x)\right|^{2} d x+2(2+\epsilon) \int_{0}^{t}\left\|u_{\tau}(\tau, \cdot)\right\|_{L_{\rho}^{2}}^{2} d \tau \\
& +\epsilon \int\left(|\nabla u(t, x)|^{2}+m^{2} \rho(x)|u(t, x)|^{2}\right) d x-2(2+\epsilon) E(0)+2 \zeta \\
\geq & (4+\epsilon) \int \rho(x)\left|u_{t}(t, x)\right|^{2} d x+2(2+\epsilon) \int_{0}^{t}\left\|u_{\tau}(\tau, \cdot)\right\|_{L_{\rho}^{2}}^{2} d \tau \\
& +\epsilon \int\left(m^{2}+\alpha\right) \rho(x)\left|u_{0}(x)\right|^{2} d x-2(2+\epsilon) E(0)+2 \zeta
\end{aligned}
$$

for every $t \in\left[0, T_{\max }\right)$.

By (22) and (23) we see that

$$
\frac{\epsilon\left(m^{2}+\alpha\right)}{2+\epsilon} \int \rho(x)\left|u_{0}(x)\right|^{2} d x-2 E(0)>0
$$

for every $m \in \mathbb{R}$.

We now let $\zeta$ satisfy

$$
0<\zeta \leq \frac{\epsilon\left(m^{2}+\alpha\right)}{2+\epsilon} \int \rho(x)\left|u_{0}(x)\right|^{2} d x-2 E(0)
$$


for every $m \in \mathbb{R}$, the other positive constants, $T_{0}$, and $T_{1}$, be large such that

$$
\begin{aligned}
& T_{0} \geq \frac{4 G(0)}{\epsilon G^{\prime}(0)}, \\
& \frac{\epsilon}{2}\left(\int \rho(x) u_{0}(x) u_{1}(x) d x+\zeta T_{1}\right)>\int \rho(x)\left|u_{0}(x)\right|^{2} d x .
\end{aligned}
$$

We next let $A, B, C$ denote the same terms as (38), (39), and (40), respectively.

We assume that the solution $u(t)$ exists on $\left[0, T_{0}\right]$. Then we have

$$
G(t) \geq A
$$

and

$$
G^{\prime \prime}(t) \geq(4+\epsilon) C
$$

for every $t \in\left[0, T_{0}\right]$.

In the same way as the proof of Theorem 2 we have

$$
G(t) G^{\prime \prime}(t)-\frac{4+\epsilon}{4}\left(G^{\prime}(t)\right)^{2} \geq(4+\epsilon)\left(A C-B^{2}\right) \geq 0 .
$$

By Lemma 2 we see that there exists a finite time $T_{\max }<\infty$ such that the corresponding solution blows up in finite time $T_{\max }$.

\section{Competing interests}

The authors declare that they have no competing interests.

Authors' contributions

Both authors contributed equality to the writing of this paper. Both authors read and approved the final manuscript.

\section{Author details}

${ }^{1}$ Institute of Applied Physics and Computational Mathematics, No. 2, Fenghaodonglu, District Haidian, Beijing, 100094,

China. ${ }^{2}$ School of Mathematics and Statistics, Wuhan University, Wuhan, 430072, China.

\section{Acknowledgements}

This work was supported by National Natural Science Foundation of China $(11371069,11671413,11001020)$. The authors would like to express their thanks to the referees for their constructive suggestion.

\section{Publisher's Note}

Springer Nature remains neutral with regard to jurisdictional claims in published maps and institutional affiliations.

Received: 22 August 2016 Accepted: 28 February 2017 Published online: 22 March 2017

\section{References}

1. Antman, SS: The equations for large vibrations of strings. Am. Math. Mon. 87, 359-370 (1980)

2. Romanov, VG: Inverse Problems of Mathematical Physics. VNU, Utrecht (1987)

3. Zauderer, E: Partial Differential Equations of Applied Mathematics, 2nd edn. Pure and Applied Mathematics. A Wiley-Intersci. Publ. Wiley, New York (1989)

4. Klibanov, MV: Global convexity in a three-dimensional inverse acoustic problem. SIAM J. Math. Anal. 28, 1371-1388 (1997)

5. Levine, HA: Instability and nonexistence of global solutions to nonlinear wave equations of the form $P u_{t t}=-A u+F(u)$. Trans. Am. Math. Soc. 192, 1-21 (1974)

6. Cazenave, T: Uniform estimates for solutions of nonlinear Klein-Gordon equations. J. Funct. Anal. 60, 36-55 (1985)

7. Levine, HA: Some additional remarks on the nonexistence of global solutions to nonlinear wave equations. SIAM J. Math. Anal. 5, 138-146 (1974)

8. Messaoudi, SA: Blow up in a nonlinearly damped wave equation. Math. Nachr. 231, 105-111 (2001)

9. Vitillaro, E: Global existence theorems for a class of evolution equations with dissipation. Arch. Ration. Mech. Anal. $149,155-182$ (1999) 
10. Levine, HA, Todorova, G: Blow up of solutions of the Cauchy problem for a wave equation with nonlinear damping and source terms and positive initial energy. Proc. Am. Math. Soc. 129, 793-805 (2001)

11. Todorova, G, Vitillaro, E: Blow-up for nonlinear dissipative wave equations in $\mathbb{R}^{n}$. J. Math. Anal. Appl. 303, 242-257 (2005)

12. Gazzola, F, Squassina, M: Global solutions and finite time blowup for damped semilinear wave equations. Ann. Inst. Henri Poincaré, Anal. Non Linéaire 23, 185-207 (2006)

13. Wang, Y: A sufficient condition for finite time blow up of the Klein-Gordon equation with arbitrarily positive initial energy. Proc. Am. Math. Soc. 136, 3477-3482 (2008)

14. Wang, Y: Non-existence of global solutions of a class of coupled non-linear Klein-Gordon with non-negative potential and arbitrary initial energy. IMA J. Appl. Math. 74, 392-415 (2009)

15. Graber, PJ, Said-Houari, B: On the wave equation with semilinear porous acoustic boundary condition. J. Differ. Equ. 252, 4898-4941 (2012)

16. Todorova, G, Yordanov, B: Critical exponent for a nonlinear wave equation with damping. J. Differ. Equ. 174, 464-489 (2001)

17. Eidus, D: The Cauchy problem for the wave equation in an inhomogeneous medium. Commun. Partial Differ. Equ. 20 1589-1603 (1995)

18. Karachalios, NI, Stavrakakis, NM: Existence of a global attractor for semilinear dissipative wave equations on $R^{N}$. J. Differ. Equ. 157, 183-205 (1999)

19. Karachalios, NI, Stavrakakis, NM: Global existence and blow-up results for some nonlinear wave equations on $\mathbb{R}^{N}$. Adv. Differ. Equ. 6, 155-174 (2001)

20. Sattinger, DH: On global solution of nonlinear hyperbolic equations. Arch. Ration. Mech. Anal. 30, 148-172 (1968)

21. Zhou, Y: Global existence and nonexistence for a nonlinear wave equation with damping and source terms. Math. Nachr. 278, 1341-1358 (2005)

22. Bilgin, BA, Kalantarov, VK: Blow up of solutions to the initial boundary value problem for quasilinear strongly damped wave equations. J. Math. Anal. Appl. 403, 89-94 (2013)

23. Kozono, H, Sohr, H: New a priori estimates for the Stokes equations in exterior domains. Indiana Univ. Math. J. 40, 1-27 (1991)

24. Brown, KJ, Stavrakakis, N: Global bifurcation results for a semilinear elliptic equation on all of $\mathbb{R}^{n}$. Duke Math. J. 85, 77-94 (1996)

25. Ono, K: On global existence, asymptotic stability and blowing up of solutions for some degenerate nonlinear wave equations of Kirchhoff type with a strong dissipation. Math. Methods Appl. Sci. 20, 151-177 (1997)

\section{Submit your manuscript to a SpringerOpen ${ }^{\circ}$ journal and benefit from:}

- Convenient online submission

Rigorous peer review

Immediate publication on acceptance

- Open access: articles freely available online

- High visibility within the field

- Retaining the copyright to your article 